Psychiatry

Elsevier Editorial system(tm) for The Lancet Manuscript Draft

Manuscript Number:

Title: Prolonged exposure to job strain and long-term mental health disorders

Article Type: Comment

Keywords: Job strain; Job demands; Job control; Demand-control support model; Work stress; Mental Health; Common Mental Disorders.

Corresponding Author: Dr. Sabir Issa Giga, Ph.D.

Corresponding Author's Institution: Lancaster University

First Author: Sabir Issa Giga, Ph.D.

Order of Authors: Sabir Issa Giga, Ph.D.

Manuscript Region of Origin: UNITED KINGDOM

Abstract: $\mathrm{N} / \mathrm{A}$ 


\section{Prolonged exposure to job strain and long-term mental health disorders}

Almost four decades ago Robert Karase ${ }^{1}$ introduced his job demands-control model highlighting a symbiotic relationship between job demands, such as the intensity and pace of work, and job control, which refers to influence and discretion in relation to personal tasks and goals. His study was based on survey data from representative random samples of adult working populations from Sweden and the United States of America. From his analysis, Karasek ${ }^{1}$ proposed four possible outcomes that could result from the interaction between job demands and job control. Much of the research carried out subsequently on this model, including the present study by Harvey and colleagues ${ }^{2}$, has focussed on the risk of elevated levels of strain that result from high demand and low control work and the impact on health and wellbeing. Karasek's ${ }^{1}$ model also suggests that high demand combined with high level of control could have positive implications and make work more challenging and motivating, low demand and high control may lead to low-strain jobs and low demand and low control is potentially linked to a passive work environment.

Whilst there is a growing body of research on the job demands-control model supporting the premise of an association between job strain and physical and mental health ${ }^{3,4,5,6}$ questions on causality continue to be raised including the possibility that pre-existing mental ill-health could lead individuals to form a negative view of their work or even be a barrier to securing a fulfilling job ${ }^{3}$. Furthermore, a broad range of other factors such as socio-demographic variables and experiences of stressful life events are also highlighted as potential factors that may negatively influence an individual's perceptions of their job and work environment.

In The Lancet Psychiatry, Samuel B Harvey and colleagues ${ }^{2}$ report the results from an analysis of data from the National Child Development Study (NCDS) in the United Kingdom ( $n=6870)$. They respond to concerns about causality and confounding by applying multivariate logistic regression to analyse the prospective association between job strain at age 45 and the risk of Common Mental Disorders (CMDs) at age 50 and controlling for a number of known confounding variables such as marital status, stressful life events and psychiatric conditions. Their results highlight a significant positive association between the odds of $C M D$ and low job control ( $O R=1.89)$, high demands $(O R=1.70)$ and high job strain (OR=2.22).

Karase ${ }^{1}$ highlighted a number of limitations relating to the research that led to the development of his model including the exclusion of female participants because it was thought in their case that 
"the relationship between work and mental health was complicated by the additional demand of housework". His model has also been criticised for relying on an individual level of analysis and ignoring broader and potentially important work group and organisational level aspects ${ }^{7}$. Although the inclusion of women has been rectified in subsequent research, the complexities of work-related stress research and interlinkages between psychological wellbeing and non-work related factors such as home and personal lives remains under-researched and are also a limitation of the present study $^{7}$. Although Harvey et al. ${ }^{2}$ consider adverse life events that have occurred in the previous 6 months, other non-work factors that may influence job strain such as caring or parental responsibilities are omitted.

Many other researchers have highlighted potential issues with relying on self-report measures to explore the relationship between job strain and mental disorders ${ }^{7}$, including common methods variance leading to the possibility of a positive or negative emotional state influencing both the reporting of strain as well as under or over-estimating the risk of CMDs. Furthermore, as Karasek's job demands-control model ${ }^{1}$ is focussed on job strain as experienced by the individual, other individual factors such as personality and coping skills, as well as group and organisational psychosocial work stressors such as resources, reward, job conflict and job security are excluded in the present study.

Although Harvey et al. ${ }^{2}$ aim to address the issue of residual confounding by systematically controlling for a number of variables throughout the life-course of participants, they highlight the need for future research to consider temporal patterns of exposure to job strain (including significant one-off events). Moreover, as day-to-day fluctuations in the severity of CMDs may influence variables as could a range of non-work factors such as personality. More meaningful questions would be required than a simple tick box question on marital status (for example, the quality of a relationship within or without marriage). Furthermore, as research suggests that there is a curvilinear relationship between job demands and outcomes such as performance and satisfaction ${ }^{4}$, sufficient attention should also be paid to exploring the impact of both prolonged high and low strain on negative health outcomes.

Notwithstanding these limitations, by working with a large sample size, adopting a multiple-point longitudinal design and controlling for confounding variables, the present research makes a significant contribution to research in this area by confirming aspects of the job demand-control model and the longer-term impact of job strain on CMDs. The findings from this research suggest 
that job strain has long-term personal, organisational and societal consequences and there is therefore a need for early interventions specifically aimed at improving employee control and involvement in decision making. The methodology adopted by Harvey et al. ${ }^{2}$ has the potential to explore other research areas such as the link between long-term high and low job strain on neurodegenerative conditions such as dementia and Parkinson's disease.

\section{Sabir I. Giga}

Division of Health Research, Faculty of Health and Medicine, Lancaster University, Lancaster, LA1 4YG, UK s.giga@lancaster.ac.uk

I declare no competing interests.

1 Karasek RA. Job demands, job decision latitude, and mental strain: Implications for job redesign. Administrative Science Quarterly 1979; 24(22): 384-408.

2 Harvey SB, Sellahewa DA, Wang M, et al. The role of job strain in understanding mid-life common mental disorder: evidence from a national birth cohort study. Lancet Psychiatry 2018; incomplete reference.

3 Henderson M, Madan I, Hotopf M. Work and mental health in the UK. BMJ 2014; 348:g2256.

4 Janssen $\mathrm{O}$. Fairness perceptions as a moderator in the curvilinear relationships between job demands, and job performance and job satisfaction. Academy of Management Journal 2001; 44(5): 1039-1050.

5 Madsen IEH, Nyberg ST, Magnusson Hanson LL. Job strain as a risk factor for clinical depression: Systematic review and meta-analysis with additional individual participant data. Psychological Medicine 2017; 47: 1342-1356.

6 Netterstrøm B. Job strain as a measure of exposure to psychological strain. Lancet Psychiatry 2012; 380: 1455-6. 
7 Burr H, Formazin M, Pohrt A. Methodological and conceptual issues regarding occupational psychosocial coronary heart disease epidemiology. Scand J Work Environ Health 2016; 42(3): 251-255. 